\title{
An Alternative Proof of Rubin's Lemma
}

\author{
Santosh Ghimire \\ Department of Applied Sciences and Chemical Engineering \\ Pulchowk Campus, Tribhuvan University, Kathmandu, Nepal \\ Email: santoshghimire@ioe.edu.np
}

\begin{abstract}
Rubin's Lemma is an inhomogenous type inequality which is satisfied by the sequence of dyadic martingales. In this paper, we give a proof using the measure theoretic approach which is simpler and different than the original probabilistic approach.
\end{abstract}

Keywords: Martingales, Law of the iterated logarithm, Quadratic characteristic, Dyadic intervals

\section{Introduction}

Rubin's Lemma is an inequality associated to a sequence of dyadic martingales and plays a vital role in the law of the iterated logarithm of dyadic martingales and harmonic functions. These law of the iterated logarithm give the asymptotic behaviour of the sequence of both functions: dyadic martingales and harmonic functions. We can study the role of Rubin's Lemma in the asymptotic behaviour of these functions in the various papers, for instances we refer a few [2] and [5]. In these papers, we find applications of the lemma in order to estimate the size of various sets in the law of the iterated logarithm. There are much more literature available related to the law of the iterated logarithm in various contexts, for instances [1], [3], [4], [6] and [8]. The proof of the Lemma can be found in [2] in which the proof uses the probabilistic approach. In the present work, we give a proof of the Lemma using the measure-theoretic approach. The original proof uses the probabilistic tools of statistics whereas our method uses the simple tools of measure theory which can be easily followed and thus the new proof is simpler in comparison with the original proof. Moreover, our method provides the proof of the lemma in the setting of mathematical analysis.

\section{Definitions and Notations}

Before proceeding with the main work, we first fix some notations and give some basic definitions which will be used in the course of proof.

A dyadic subinterval of the unit interval $[0,1]$ is an interval of the form $\left[\frac{j}{2^{n}}, \frac{j+1}{2^{n}}\right)$ where $n=0,1,2, \ldots$ and $j=0,1, \ldots, 2^{n}-1$.

A dyadic martingale is a sequence of integrable functions $\left\{f_{n}\right\}_{n=0}^{\infty}$ where $f_{n}:[0,1) \rightarrow \mathbb{R}$ such that

a. for every $n, f_{n}$ is $f_{n}$ measurable where $f_{n}$ is the sigma algebra generated by the dyadic intervals of the form $\left[\frac{j}{2^{n}}, \frac{j+1}{2^{n}}\right)$ for $j=0,1, \ldots, 2^{n}-1$.

b. conditional expectation $E\left(f_{n+1} \mid f_{n}\right)=f_{n}$ where

$$
E\left(\mathrm{f}_{\mathrm{n}+1} \mid f_{n}\right)(x)=\frac{1}{|Q|} \int_{Q_{n}} f_{n}(y) d y \text { with }\left|Q_{n}\right|=\frac{1}{2^{n}}, x \in Q_{n} .
$$


Associated with a sequence of dyadic martingales $\left\{f_{n}\right\}_{n=0}^{\infty}$, we define:

i. increments: $d_{k}=f_{k}-f_{k+1}$. So $f_{n}(x)=\sum_{k=1}^{n} d_{k}(x)+f_{0}$.

ii. quadratic characteristics or square function: $S_{n}^{2} f(x)=\sum_{k=1}^{n} d_{k}^{2}(x)$.

iii. $\quad$ limit function $S^{2} f(x)=\lim _{n \rightarrow \infty} S_{n}^{2} f(x)=\sum_{k=1}^{\infty} d_{k}^{2}(x)$.

We now state the inequality associated to the dyadic martingales and this inequality is called Rubin's Lemma according to [7].

Rubin's Lemma: For a dyadic martingales $\left\{f_{n}\right\}_{n=0}^{\infty}$ with $f_{0}=0$, we have

$$
\int_{0}^{1} \exp \left(f_{n}(x)-\frac{1}{2} S_{n}^{2} f(x)\right) d x \leq 1 \text {. }
$$

We now give the proof the lemma using a different approach than the original proof.

\section{Proof of the Main Result}

Let us first define

$$
g(n)=\int_{0}^{1} \exp \left(\sum_{k=0}^{n} d_{k}(x)-\frac{1}{2} \sum_{k=0}^{n} d_{k}^{2}(x)\right) d x
$$

We now claim that the function $g(n)$ is a decreasing function of $n$. Let $Q_{n j}$ be an arbitrary $n^{\text {th }}$ generation dyadic interval. One can see that $\sum_{k=0}^{n} d_{k}(x)=f_{n}$ and $f_{n}$ is constant on $Q_{n j}$.

Using this fact, we have

$$
\begin{aligned}
g(n+1) & =\sum_{j=0}^{2^{n}} \int_{Q_{n j}} \exp \left(\sum_{k=0}^{n+1} d_{k}(x)-\frac{1}{2} \sum_{k=0}^{n+1} d_{k}^{2}(x)\right) d x \\
& =\sum_{j=0}^{2^{n}} \int_{Q_{n j}} \exp \left(\sum_{k=0}^{n} d_{k}(x)-\frac{1}{2} \sum_{k=0}^{n} d_{k}^{2}(x)\right) \exp \left(d_{n+1}(x)-\frac{1}{2} d_{n+1}^{2}(x)\right) d x . \\
& =\sum_{j=0}^{2^{n}}\left[\exp \left(\sum_{k=0}^{n} d_{k}(x)-\frac{1}{2} \sum_{k=0}^{n} d_{k}^{2}(x)\right)\right]_{Q_{n j}} \int_{Q_{n j}} \exp \left(d_{n+1}(x)-\frac{1}{2} d_{n+1}^{2}(x)\right)
\end{aligned}
$$

Let $Q_{(n+1) j}^{\prime}$ and $Q_{(n+1) j}^{\prime \prime}$ be the dyadic subintervals of $Q_{n j}$. Suppose $d_{n+1}$ takes the value $\alpha$ on $Q_{(n+1) j}^{\prime}$.

Then by expectation condition, $d_{n+1}$ takes the value $-\alpha$ on $Q_{(n+1) j}^{\prime \prime}$.

This gives

$$
\begin{aligned}
\int_{Q_{n j}} \exp \left(d_{n+1}(x)-\frac{1}{2} d_{n+1}^{2}(x)\right) & \\
= & \int_{Q_{(n+1) j}^{\prime}} \exp \left(d_{n+1}(x)-\frac{1}{2} d_{n+1}^{2}(x)\right) d x+\int_{Q_{(n+1) j}^{\prime \prime}} \exp \left(d_{n+1}(x)-\frac{1}{2} d_{n+1}^{2}(x)\right) d x
\end{aligned}
$$




$$
\begin{aligned}
& =\int_{Q_{(n+1) j}^{\prime}} \exp \left(\alpha-\frac{1}{2} \alpha^{2}\right) d x+\int_{Q_{(n+1) j}^{\prime \prime}} \exp \left(-\alpha-\frac{1}{2} \alpha^{2}\right) d x \\
& =\left[\exp \left(\alpha-\frac{1}{2} \alpha^{2}\right)+\exp \left(-\alpha-\frac{1}{2} \alpha^{2}\right)\right] \frac{1}{2^{n+1}} \\
& =2 \exp \left(-\frac{\alpha^{2}}{2}\right)\left(\frac{e^{\alpha}+e^{-\alpha}}{2}\right) \frac{1}{2^{n+1}}
\end{aligned}
$$

Thus, $\int_{Q_{n j}} \exp \left(d_{n+1}(x)-\frac{1}{2} d_{n+1}^{2}(x)\right)=2 \exp \left(-\frac{\alpha^{2}}{2}\right) \cosh \alpha \frac{1}{2^{n+1}}$.

Now using the elementary fact that $\cosh x \leq \exp \left(\frac{x^{2}}{2}\right)$,we have

$$
\begin{aligned}
& g(n+1) \leq \sum_{j=0}^{2^{n}}\left[\exp \left(\sum_{k=0}^{n} d_{k}(x)-\frac{1}{2} \sum_{k=0}^{n} d_{k}^{2}(x)\right)\right]_{Q_{n j}} 2 \exp \left(-\frac{\alpha^{2}}{2}\right) \exp \left(\frac{\alpha^{2}}{2}\right) \frac{1}{2^{n+1}} \\
& g(n+1) \leq \sum_{j=0}^{2^{n}}\left[\exp \left(\sum_{k=0}^{n} d_{k}(x)-\frac{1}{2} \sum_{k=0}^{n} d_{k}^{2}(x)\right)\right]_{Q_{n j}}\left|Q_{n j}\right| \\
& g(n+1) \leq \sum_{j=0}^{2^{n}} \int_{Q_{n j}} \exp \left(\sum_{k=0}^{n} d_{k}(x)-\frac{1}{2} \sum_{k=0}^{n} d_{k}^{2}(x)\right) d x \\
& g(n+1) \leq g(n) .
\end{aligned}
$$

Let $Q_{11}$ and $Q_{12}$ be the dyadic subintervals of $Q_{0}$. Assume that $d_{1}$ takes value $\theta$ on $Q_{11}$ so that it takes value $-\theta$ on $Q_{12}$.

Now $g(1)=\int_{0}^{1} \exp \left(d_{1}(x)-\frac{1}{2} d_{1}^{2}(x)\right) d x$

$$
\begin{aligned}
& =\int_{0}^{\frac{1}{2}} \exp \left(\theta-\frac{1}{2} \theta^{2}\right) d x+\int_{\frac{1}{2}}^{1} \exp \left(-\theta-\frac{1}{2} \theta^{2}\right) d x \\
& =\exp \left(\theta-\frac{1}{2} \theta^{2}\right) \frac{1}{2}+\exp \left(-\theta-\frac{1}{2} \theta^{2}\right) \frac{1}{2} \\
& =\exp \left(-\frac{1}{2} \theta^{2}\right) \frac{\left(e^{\theta}+e^{-\theta}\right)}{2} \\
& =\exp \left(-\frac{1}{2} \theta^{2}\right) \cosh \theta \\
& g(1) \leq \exp \left(-\frac{1}{2} \theta^{2}\right) \exp \left(\frac{1}{2} \theta^{2}\right)=1 .
\end{aligned}
$$

Since $g(n)$ is decreasing and $g(1) \leq 1$, we conclude that $g(n) \leq 1$.

This gives

$$
\int_{0}^{1} \exp \left(\sum_{k=0}^{n} d_{k}(x)-\frac{1}{2} \sum_{k=0}^{n} d_{k}^{2}(x)\right) d x \leq 1
$$


Hence, $\int_{0}^{1} \exp \left(f_{n}(x)-\frac{1}{2} S_{n}^{2} f(x)\right) d x \leq 1$.

This proves our result.

Finally we note that if we rescale the function by some $\lambda$, we have

$$
\int_{0}^{1} \exp \left(\lambda f_{n}(x)-\frac{1}{2} \lambda^{2} S_{n}^{2} f(x)\right) d x \leq 1 .
$$

This shows that the Rubin's Lemma is an inhomogeneous type inequality.

\section{Conclusion}

In this paper, we derived an inequality associated to a sequence of dyadic martingales which is popularly known as Rubin's Lemma. We used the measure theoretic approach to prove the inequality which is different than the original probabilistic approach.

\section{Acknowledgement}

The author would like to thank the referees for valuable insightful comments that helped to improve the paper.

\section{References}

[1] Benelos R. and Moore C.N. (1991). Probabilistic Behavior of Harmonic Functions, Birkhauser Verlag.

[2] Chang S. Y., Wilson J.M. and Wolff T.H. (1985). Some weighted norm inequalities concerning the Schrodinger operator, Commentarii Mathematici Helvetici, 60: 217-246.

[3] Ghimire S. and Moore C. N. (2014). A lower bound in the tail law of the iterated logarithm of lacunary trigonometric series, Proceeding of American Mathematical Society, 142: 3207-3216.

[4] Ghimire S. (2014). The law of the iterated logarithm in analysis, Journal of Institute of Engineering, 9: 89-94.

[5] Moore C. N. (2018). Subgaussian estimates in probability and harmonic analysis, Journal of Analysis 26:175-190.

[6] Petrov V. V . (2020). The law of the iterated logarithm and probabilities of moderate deviations of sums of dependent random variables, Journal of Mathematical Sciences, 251: 128-130.

[7] Pipheer J. (1993). A martingale inequality related to exponential square integrability, Proceeding of the American Mathematical Society, 118(2): 541-546.

[8] Stout W. (1970). A martingale analogue of Kolmogorov's law of the iterated logarithm, Z. Wahrscheinlichkeitsthorie Verw. Geb, 15: 279-290. 